\title{
DECOMPOSITION OF NORMAL CURRENTS
}

\author{
MACIEJ ZWORSKI
}

(Communicated by David G. Ebin)

\begin{abstract}
As an answer to a question of Frank Morgan, it is shown that there exist normal currents which cannot be represented as convex integrals of rectifiable currents. However, under certain additional hypotheses, such decompositions exist. Examples are given to indicate that such hypotheses are necessary.
\end{abstract}

1. Introduction. ${ }^{1}$ The purpose of this note is to answer a question $(*)$ posed by Frank Morgan (compare Problem 3.8 in [B]):

Does every normal current in codimension one admit a nice mass decomposition as an integral of rectifiable currents?

Specifically for $m=n-1$ and $N \in \mathbf{N}_{m}\left(\mathbf{R}^{n}\right)$ does there exist a family $\left\{R_{\omega}\right\}_{\omega \in \Omega}$ such that

$$
\begin{aligned}
& R_{\omega} \in \mathbf{R}_{m}\left(\mathbb{R}^{n}\right) \text { and } \Omega \text { is some measure space, } \\
& N=\int_{\Omega} R_{\omega} d \omega \\
& \|N\|=\int_{\Omega}\left\|R_{\omega}\right\| d \omega \\
& \|\partial N\|=\int_{\Omega}\left\|\partial R_{\omega}\right\| d \omega
\end{aligned}
$$

Condition (4) means that the mass decomposition is simultaneously a boundary mass decomposition.

This problem has been first studied by Fleming and Rishel [FR] (see also [F, 4.9.15(13)]), who proved existence of a decomposition satisfying (1)-(4) for currents $N \in \mathbb{N}_{n-1}\left(\mathbb{R}^{n}\right)$ with $\partial N=0$. In this case $N=\partial\left(\mathbb{E}^{n} L f\right)$ and

$$
\begin{array}{r}
N=\int \partial\left(\mathbb{E}^{n} L\{x: f(x) \geq s\}\right) d L_{s}^{1}, \\
\|N\|=\int\left\|\partial\left(\mathbb{E}^{n} L\{x: f(x) \geq s\}\right)\right\| d L_{s}^{1} .
\end{array}
$$

As remarked by Morgan this also implies decomposition in the case of extremal boundary.

Recently Hardt and Pitts [HP] solved Plateau's Problem for hypersurfaces using an ingeneous modification of the level set argument (5) from [FR]. Their proof shows the existence of a decomposition satisfying (1)-(4) in the case of $N \in \mathbb{N}_{n-1}\left(\mathbb{R}^{n}\right)$ with $\partial N \in \mathbf{R}_{n-2}\left(\mathbb{R}^{n}\right)$ (Theorem 1 ).

Received by the editors January 3, 1987.

1980 Mathematics Subject Classification (1985 Revision). Primary 49F20; Secondary 53C12.

${ }^{1}$ The notation throughout the paper is taken from [F]. 
Despite these facts, the question $(*)$ has a negative answer: there exists a large class of currents which do not have any mass decomposition, i.e., a decomposition satisfying even just (1)-(3). This is the main result of the paper and it is contained in the necessity part of Theorem 2 . Theorem 2 also provides some positive results about smooth normal currents in any codimension. Unlike in codimension one, decompositions in higher codimensions are simultaneous boundary mass decompositions only locally and the smoothness assumption cannot be dropped (Proposition 3 ). Finally, even if a nonsmooth current admits a mass decomposition it may have no simultaneous boundary mass decomposition, even locally (Proposition 2).

I should like to thank Professor Frank Morgan for his invaluable advice and constant encouragement during the preparation of this paper.

2. Hardt-Pitts decomposition. We are going to outline the proof of the following observation based on [HP].

THEOREM 1. If $N \in \mathbb{N}_{n-1}\left(\mathbb{R}^{n}\right)$ and $\partial N \in \mathbf{R}_{n-2}\left(\mathbb{R}^{n}\right)$, then there exists a family $\left\{R_{\omega}\right\}_{\omega \in \Omega}$ such that $(1)-(4)$ hold, with $\Omega=(0,1)$.

OUtline OF THE PROOF. Let $R, \lambda, \mu$ be as in [HP]. Then for almost all $s \in(0,1)$ and all $j \in \mathbb{Z}$,

$$
\|R\|\left(\mu^{-1}(s+j) \cup \lambda^{-1}(s+j)\right)=0 .
$$

Hence, we can define

$$
R_{s}=R-\sum_{j \in \mathbb{Z}} \partial\left[\mathbb{E}^{n} L\{x: f(x) \geq s+j\}\right]
$$

and, as in $[\mathbf{H P}]$,

$$
\partial R_{s}=\partial N, \quad\left\|\sum_{j \in \mathbb{Z}} \partial\left[\mathbb{E}^{n} L\{f \geq s+j\}\right]\right\|=\|R\|+\left\|R_{s}\right\|,
$$

which by the choice of $R$ and (6) implies (1)-(4) with $\Omega=(0,1)$.

REMARK. Since the above construction gives $\partial R_{s}=\partial N$, for all $s$, it follows that if $N$ is area minimizing, so are almost all of $R_{s}$. That was the method of proving the existence of area minimizing hypersurfaces in [HP]. Also Morgan's Regularity Theorem for normal area minimizing currents [M, Theorem 9.1$]$ can be easily deduced from Theorem 1 .

3. Decompositions and integrability. A normal current $N \in \mathbb{N}_{m}\left(\mathbb{R}^{n}\right)$ is called smooth if there exists a $C^{\infty} m$-vectorfield, such that $N=L^{n} \wedge \xi$. The current is called simple if $\xi$ is simple, i.e. $\xi=\eta_{1} \wedge \cdots \wedge \eta_{m}$ for some $C^{\infty}$ vectorfields $\eta_{i}$. By integrability of $\xi$ we mean integrability of the distribution given by $\xi$ in the sense of the Frobenius theorem.

For smooth currents the notion of mass decomposition is closely related to the integrability of $\xi$, and we have

THEOREM 2. Let $N \in \mathbb{N}_{m}\left(\mathbb{R}^{n}\right)$ be smooth and simple, $N=L^{n} \wedge \xi$. Then $N$ has a decomposition satisfying (1) (3) if and only if the vectorfield $\xi$ is integrable. Moreover, if $\xi$ is integrable then for every $x \in \operatorname{int}(\operatorname{spt} N)$ there exists a neighbourhood 
$U, x \in U$, and a family $\left\{R_{s}\right\}_{s \in \mathbf{R}^{n-m+1}}$ such that

$$
\begin{gathered}
R_{s} \in \mathbf{R}_{m}\left(\mathbb{R}^{n}\right), \quad \text { for } s \in \mathbb{R}^{n-m+1}, \\
N L U=\int R_{s} d L_{s}^{n-m+1}, \\
\|N L U\|=\int\left\|R_{s}\right\| d L_{s}^{n-m+1}, \\
\|\partial N L U\|=\int\left\|\partial R_{s} L U\right\| d L_{s}^{n-m+1} . a
\end{gathered}
$$

REMARK. This theorem provides a large class of normal currents without any rectifiable mass decomposition-namely those which are given by nonintegrable vectorfields. In the case of integrable vectorfields we get local simultaneous boundary mass decompositions. Even in codimension one the decomposition has to be, in general, local and the smoothness assumption is essential (see §4).

For the proof of the theorems we shall need some lemmas.

LEMMA A. For a simple $m$-vectorfield $\xi$ of class $2, \xi=\eta_{1} \wedge \cdots \wedge \eta_{m}$, the following conditions are equivalent:

(11) $\xi$ is integrable.

(12) $\operatorname{div} \xi \wedge \eta_{i} \wedge \eta_{j}=0$ for all $1 \leq i, j \leq m$,

(13) $\operatorname{div} \xi=\sum \phi_{j}\left(\eta_{1} \wedge \cdots \wedge \eta_{j-1} \wedge \eta_{j+1} \wedge \cdots \wedge \eta_{m}\right)$ for some functions $\phi_{j} \in C^{1}$.

PROOF. By the Frobenius theorem the first condition is equivalent to

$$
\left[\eta_{i}, \eta_{j}\right] \in \operatorname{span}\left\{\eta_{1}, \ldots, \eta_{m}\right\} \quad \text { for all } 1 \leq i, j \leq m \text {. }
$$

Define $\mathbf{D}_{m} \xi=\xi L\left(D X_{1} \wedge \cdots \wedge D X_{n}\right)$, and we have $[\mathbf{F}, 4.1 .6]$

$$
d\left(\mathbf{D}_{m} \xi\right)=(-1)^{n-m} \mathbf{D}_{m-1}(\operatorname{div} \xi)
$$

Also [F, 4.1.34]

(16)

$$
\begin{aligned}
\left\langle\xi_{1} \wedge \cdots \wedge \xi_{n-m+1}, d\left(\mathbf{D}_{n} \xi\right)\right\rangle= & \sum_{r=1}^{n-m+1}(-1)^{r-1}\left\langle\xi_{r}, D\left(\xi^{(r)}, \mathbf{D}_{m} \xi\right)\right\rangle \\
& +\sum_{r=1}^{n-m+1} \sum_{s=r+1}^{n-m+1}(-1)^{r-1}\left\langle\left[\xi_{r}, \xi_{s}\right] \wedge \xi^{(r, s)}, \mathbf{D}_{m} \xi\right\rangle
\end{aligned}
$$

where

$$
\begin{aligned}
& \xi^{(r)}=\xi_{1} \wedge \cdots \wedge \xi_{r-1} \wedge \xi_{r+1} \wedge \cdots \wedge \xi_{n-m+1} \\
& \xi^{(r, s)}=\xi_{1} \wedge \cdots \wedge \xi_{r-1} \wedge \xi_{r+1} \wedge \cdots \wedge \xi_{s-1} \wedge \xi_{s+1} \wedge \cdots \wedge \xi_{n-m+1}
\end{aligned}
$$

We can apply (16) with $\xi_{1}=\eta_{i}, \xi_{2}=n_{j}, \xi_{n+2}=e_{\sigma(n)}, r \leq n-m-1, \sigma \in \mathbf{S}_{n}$. Since $\xi^{(r)}=\eta_{i} \wedge \tau$ or $\xi^{(r)}=\eta_{j} \wedge \tau$ for some $\tau$, we obtain $\left\langle\xi^{(r)}, \mathbf{D}_{m} \xi\right\rangle=0$ for

$$
\left\langle\eta_{i} \wedge \tau, \mathbf{D}_{m} \xi\right\rangle=\left\langle\eta_{i} \wedge \tau \wedge \eta_{1} \wedge \cdots \wedge \eta_{m}, D X_{1} \wedge \cdots \wedge D X_{n}\right\rangle=0 .
$$

Similarly, $\left\langle\left[\xi_{r}, \xi_{s}\right] \wedge \xi^{(r, s)}, \mathbf{D}_{m} \xi\right\rangle=0$, unless $(r, s)=(1,2)$. Hence the right-hand side of $(16)$ is

$$
\begin{aligned}
& \left\langle\left[\eta_{i}, \eta_{j}\right] \wedge e_{\sigma(1)} \wedge \cdots \wedge e_{\sigma(n-m+1)}, \mathbf{D}_{m} \xi\right\rangle \\
& \quad=\left\langle\left[\eta_{i}, \eta_{j}\right] \wedge \eta_{1} \wedge \cdots \wedge \eta_{m} \wedge e_{\sigma(1)} \wedge \cdots \wedge e_{\sigma(n-m+1)}, D X_{1} \wedge \cdots \wedge D X_{n}\right\rangle
\end{aligned}
$$


This is equal to zero for all $\sigma \in \mathbf{S}_{n}$ if and only if (14) holds. On the other hand, the left-hand side of (16) is

$$
(-1)^{n-m}\left\langle\eta_{i} \wedge \eta_{j} \wedge e_{\sigma(1)} \wedge \cdots \wedge e_{\sigma(n-m+1)} \wedge \operatorname{div} \xi, D X_{1} \wedge \cdots \wedge D X_{n}\right\rangle
$$

which is equal to zero for all $\sigma \in \mathbf{S}_{n}$ if and only if (13) is true. Also, (14) clearly implies (11). This follows from (13), since at every point where $\xi \neq 0, \eta_{1}, \ldots, \eta_{m}$, $\tilde{\eta}_{m+1}, \ldots, \tilde{\eta}_{n}$ span $\mathbb{R}^{n}$ for some $\tilde{\eta}_{j}, m<j \leq n$, and if $\operatorname{div} \xi$ had a nonzero component in the direction of $\eta_{\sigma(1)} \wedge \cdots \wedge \eta_{\sigma(m)} \tilde{\eta}_{\tilde{\sigma}(1)+m} \wedge \cdots$, and $\sigma(k)$ is not $i$ or $j$ for all $k$, $0<k<m+1$, then $\operatorname{div} \xi \wedge \eta_{i} \wedge \eta_{j} \neq 0$.

We shall need the following easy fact:

LEMMA B. If $f \in L^{1}\left(\mathbb{R}^{n}\right) \cap C^{1}\left(\mathbb{R}^{n}\right)$ and $f \geq 0$, then there exists a function $F: \mathbb{R}^{n} \rightarrow \mathbb{R}^{n}$ such that $f=\mathbf{J}_{n} F$.

Proof. Just define

$$
F\left(x_{1}, \ldots, x_{n}\right)=\int_{0}^{x_{1}} f\left(t, x_{2}, \ldots, x_{n}\right) d t .
$$

Before proving Theorem 2, we shall give its analogue in the case of no boundary:

Proposition 1. Let $N \in \mathbb{N}_{m}\left(\mathbb{R}^{n}\right)$ be smooth and simple, and also let $\partial N=0$. Then for every $x \in \operatorname{int}(\operatorname{spt} N)$ there exists a family $\left\{R_{s}\right\}_{s \in \mathbf{R}^{n-m}}$ and a neighborhood $U, x \in U$, such that

$$
\begin{aligned}
& R_{s} \in \mathbf{R}_{m}\left(\mathbb{R}^{n}\right), \\
& N\left\llcorner U=\int R_{s} d L_{s}^{n-m},\right. \\
& \| N\left\llcorner U\left\|=\int\right\| R_{s} \| d L_{s}^{n-m},\right. \\
& \partial R_{s}\llcorner U=0,
\end{aligned}
$$

where spt $R_{s}$ is an integral manifold of $\xi$ with multiplicity 1.

ProOF. Since $\partial N=-L^{n} \wedge \operatorname{div} \xi$, we get $\operatorname{div} \xi=0$, which by Lemma $\mathrm{A}$ implies that $\xi$ is integrable. Hence every $x \in \operatorname{int}(\operatorname{spt} N)$ has a neighborhood $U$, such that there exists a function $G: U \rightarrow] 0,1\left[^{n}\right.$ with integral manifolds of $G_{\#} \xi$ given by

$$
\left.M_{c}=\right] 0,1\left[^{m} \times c, \quad c \in\right] 0,1\left[^{n-m}\right. \text {. }
$$

We have

$$
G_{\#} N=L^{n} \wedge G_{\#} \xi / \mathbf{J}_{n} G, \quad \partial G_{\#} N=G_{\#} \partial N=0 .
$$

It follows from (21) that $\left|G_{\#} \xi\right| / \mathrm{J}_{n} G$ has to be constant on each integral manifold, $M_{c}$. This defines a function on $] 0,1\left[{ }^{n-m}\right.$, which we shall call $a(c)$. With this notation we get

$$
G_{\#} N(\phi)=\int_{] 0,1\left[^{n-m}\right.} \int_{\mid 0,1\left[^{m} \times c\right.}\left\langle\frac{G_{\#} \xi}{\left|G_{\#} \xi\right|}, \phi\right\rangle a(c) d L^{m} d L_{c}^{n-m} .
$$

By Lemma $\mathrm{B}$ there exists a mapping $A: \mathbb{R}^{n-m} \rightarrow \mathbb{R}^{n-m}$ such that $\mathbf{J}_{n-m} A=a$. Using this, from (22) we obtain

$$
G_{\#} N(\phi)=\int_{A(] 0,1\left[^{n-m}\right)} \int_{] 0,1\left[^{m} \times A^{-1}(s)\right.}\left\langle\frac{G_{\#} \xi}{\left|G_{\#} \xi\right|}, \phi\right\rangle d L^{m} d L_{s}^{n-m}
$$


It is now easy to verify that

$$
\begin{gathered}
R_{s}=\left(G^{-1}\right)_{\#}\left(\mathbf{H}^{m}\left\llcorner(] 0,1\left[^{m} \times A^{-1}(s)\right) \wedge G_{\#} \xi /\left|G_{\#} \xi\right|\right) \quad \text { for } s \in A(] 0,1\left[^{n-m}\right),\right. \\
R_{s}=0 \text { for } s \in\left(A(] 0,1\left[^{n-m}\right)\right)^{c}
\end{gathered}
$$

satisfy (17)-(18).

PROOF OF THEOREM 2. For the proof of sufficiency we can use Proposition 1 and decompose $\partial N \in \mathbb{N}_{m-1}\left(\mathbb{R}^{n}\right)$ :

$$
\partial N L U=\int \tilde{R}_{s} d L_{s}^{n-m+1},
$$

where supports of currents $\tilde{R}_{s}$ are integral manifolds of $\xi$. If $M$ is a component of an integral manifold of $\xi$ in $U$ and spt $R_{s} \subset M$ then spt $R_{s}$ divides $M$ into disjoint sets. Let $M_{s}$ be the one which oriented by $\xi$ has spt $\tilde{R}_{s}$ oriented by $\tilde{R}_{s}$ as its boundary, and define

$$
R_{s}^{\prime}=\mathbf{H}^{m}\left\llcorner M_{s} \wedge \xi /|\xi|\right.
$$

If $N^{\prime}=\int R_{s}^{\prime} d L_{s}^{n-m+1}$, then

$$
\|\partial N L U\|=\left\|\partial N^{\prime} L U\right\|=\int\left\|\partial R_{s}^{\prime} L U\right\| d l_{s}^{n-m+1}
$$

by the choice of $\tilde{R}_{s}=\partial R_{s}^{\prime}$. Also, $\partial\left(N-N^{\prime}\right)\left\lfloor U=0\right.$ and $N-N^{\prime}$ is smooth in $U$. By Proposition 1

$$
\left(N-N^{\prime}\right)\left\llcorner U=\int T_{t} d L_{t}^{n-m}, \quad \partial T_{t} L U=0 .\right.
$$

Putting

$$
T_{(t, r)}= \begin{cases}0 & \text { for } r \in[0,1]^{c} \\ T_{t} & \text { for } r \in[0,1]\end{cases}
$$

we obtain that

$$
R_{s}=R_{s}^{\prime}+T_{s}, \quad s \in \mathbb{R}^{n-m+1},
$$

is the desired decomposition.

To obtain a global mass decomposition we can cover int(spt $\xi) L^{n}$-almost everywhere by a countable number of disjoint sets $W_{\alpha}$ such that int $W_{\alpha}$ has properties as $U$ above. This is possible thanks to $[\mathbf{F}, 2.8 .14]$ and the fact that sets $U$ can have arbitrarily small radii. Hence we can take $\Omega=\mathbb{R}^{n-m+1} \times \mathbb{Z}$ with $\Omega=\{\omega=(s, \alpha)\}$, where $N$ Lint $W_{\alpha}=\int R_{s} d L_{s}^{n-m+1}$.

To prove necessity we shall need one more lemma:

Lemma C. Let $N \in \mathbb{N}_{m}\left(\mathbb{R}_{n}\right)$ be smooth, $N=L^{n} \wedge \xi, \xi=\eta_{1} \wedge \cdots \wedge \eta_{n}$. Suppose also that $N$ has a decomposition $\left\{R_{\omega}\right\}_{\omega \in \Omega}$ satisfying (1)-(3). Then for $d \omega$-almost all $\omega$ : if $\operatorname{Tan}^{m}\left(R_{\omega}, x\right) \neq 0$, then $\operatorname{Tan}^{m}\left(R_{\omega}, x\right)$ is an $m$-plane associated with $\eta_{1} \wedge \cdots \wedge \eta_{m}$, for $\mathbf{H}^{m}$-almost all $x$.

PROOF. Suppose not, $R_{\omega}=\mathbf{H}^{m}\left\llcorner B_{\omega} \wedge \vec{R}_{\omega} \wedge \vec{R}_{\omega}\right.$ and $\vec{R}_{\omega}(x)$ has a nonzero component in a direction other than $\eta_{1} \wedge \cdots \wedge \eta_{m}$ :

$$
\vec{R}_{\omega}(x)=f_{\omega}(x) \eta_{1} \wedge \cdots \wedge \eta_{m}+\vec{E}_{\omega}(x),
$$


where $\vec{E}_{\omega} \neq 0$ and it is orthogonal to $\eta_{1} \wedge \cdots \wedge \eta_{m}(x)$. If $R^{\prime} \omega=\mathbf{H}^{m} \mathrm{~L} B \wedge f_{\omega}(x) \eta_{1} \wedge$ $\cdots \wedge \eta_{m}(x)$, then

$$
N=\int_{\Omega} R_{\omega}^{\prime} d \omega, \quad\|N\|=\int_{\Omega}\left\|R_{\omega}^{\prime}\right\| d \omega
$$

and $\left\|R_{\omega}^{\prime}\right\|<\left\|R_{\omega}\right\|$, since the set of $x$ for which $\vec{E}_{\omega}(x) \neq 0$ has a positive $\mathbf{H}^{m}$. measure. But this occurs for positive $d \omega$ measure of $\omega \in \Omega$ and hence $\|N\|<$ $\int_{\Omega}\left\|R_{\omega}\right\| d \omega$, a contradiction completing proof of the lemma.

To prove necessity in Theorem 2, suppose that $\xi$ is not integrable. It follows from Lemma $\mathrm{A}$ that $\operatorname{div} \xi(x)$ has components orthogonal to any $\eta_{1} \wedge \cdots \wedge \hat{\eta}_{i} \wedge \cdots \wedge \eta_{m}(x)$, $1 \leq i \leq m$, for $x$ in some open set $V$.

Let $\phi$ be a $(m-1)$-form with support in $V$ such that its dual is associated with the $(m-1)$-plane orthogonal to $\eta_{1} \wedge \cdots \wedge \hat{\eta}_{i} \wedge \cdots \wedge \eta_{n}$ on which $\operatorname{div} \xi$ has nonzero components. Then $\partial N(\phi) \neq 0$ since $\partial N=-L^{n} \wedge \operatorname{div} \xi$. If $R_{\omega}=\mathbf{H}^{m}\left\llcorner B_{\omega} \wedge \vec{R}_{\omega}\right.$, then using $[\mathbf{F}, 3.2 .29]$ and possibly changing the measure space we can assume that $B_{\omega}$ is a subset of $C^{1}$-manifold $M$ and by Lemma C, $\vec{R}_{\omega}=f_{\omega} \eta_{1} \wedge \cdots \wedge \eta_{m}$. Since $\xi$ is smooth, the sets of points of $M_{\omega}, A_{\omega}$ at which the tangent plane to $M_{\omega}$ is associated with $\xi$ is closed, and spt $R_{\omega} \subset A_{\omega}$.

As $\partial R_{\omega} \in \mathbb{F}_{m}\left(\mathbb{R}^{n}\right)$, we infer from the Federer Flatness Theorem [F, 4.1.15] (see also [H, II.5.2]) that

$$
\partial R_{\omega}=\sum_{j=1}^{m} T_{j}^{\omega} \eta_{i} \wedge \cdots \wedge \hat{\eta}_{j} \wedge \cdots \wedge \eta_{m}
$$

where $T_{j}^{\omega} \in \mathbf{D}^{\prime}(U), U$ open, $U \subset \operatorname{spt} N$, and the notation means that

$$
\partial R_{\omega}(\psi)=\sum_{j=1}^{m} T_{j}^{\omega}\left(\left\langle\eta_{1} \wedge \cdots \wedge \hat{\eta}_{j} \wedge \cdots \wedge \eta_{m}, \psi\right\rangle\right) .
$$

From this we obtain

$$
\begin{aligned}
\partial N & =\int_{\Omega} \partial R_{\omega} d \omega=\int \sum_{j=1}^{m} T_{j}^{\omega} \eta_{1} \wedge \cdots \wedge \hat{\eta}_{j} \wedge \cdots \wedge \eta_{m} d \omega \\
& =\sum_{j=1}^{m}\left(\int T_{j}^{\omega} d \omega\right) \eta_{1} \wedge \cdots \wedge \hat{\eta}_{j} \wedge \cdots \wedge \eta_{m} .
\end{aligned}
$$

Since $\mathbf{M}(\partial N)<\infty$, it follows that each distribution $\int_{\omega} T_{j}^{\omega} d \omega$ is a measure and, by the choice of $\phi, \partial N(\phi)=0$. This, however, gives a contradiction and proves Theorem 2.

4. Examples. In this section we shall show that the hypotheses of Theorem 2 are necessary. By Theorem 2 we get, for integrable vector fields, a local decomposition of smooth normal currents. Even in codimension one it has to be in general local, as shown by the standard example of a constant vectorfield on the torus in $\mathbb{R}^{3}$ with irrational slope and nonperiodic integral curves. Smoothing this vectorfield gives a normal current of codimension one, with no boundary and no decomposition $\left\{R_{s}\right\}_{s \in \mathbf{R}}$ such that $\|N\|=\int\left\|R_{s}\right\| d L_{s}^{2}, R_{s} \in \mathbf{R}_{1}\left(\mathbb{R}^{3}\right)$ and $\partial R_{s}=0$ for almost all $s$.

A nonsmooth normal current may have a mass decomposition, but no simultaneous boundary mass decomposition, even locally and in codimension one. 
PROPOSITION 2. There exists a current $N \in \mathbb{N}_{2}\left(\mathbb{R}^{3}\right)$ and a set $X, \mathbf{H}^{2}(X)>0$, such that no $x \in X$ has an open neighbourhood $U$ for which there exists a decomposition $\left\{R_{s}\right\}_{s \in \mathbf{R}}$ satisfying (7)-(10) $(n=3, m=2)$.

For the proof we shall need the following lemma, which in fact is the uniqueness statement for Proposition 1.

LEMMA D. Let $\eta$ be a smooth $L^{n}$-locally summable $m$-vectorfield in $\mathbf{R}^{n}, U$ an open set, and $\left\{R_{\omega}\right\}_{\omega \in \Omega}$ a family of currents such that

$$
\begin{aligned}
& R_{\omega} \in \mathbf{R}_{n}\left(\mathbb{R}^{n}\right), \\
& \left(L^{n} \wedge \eta\right)\left\llcorner U=\int_{\Omega} R_{\omega} d \omega,\right. \\
& \|\left(L^{n} \wedge \eta\right)\left\llcorner U\left\|=\int_{\Omega}\right\| R_{\omega} \| d \omega,\right. \\
& \partial R_{\omega} L U=0 .
\end{aligned}
$$

Then spt $R_{\omega} L U$ is a countable union of components of integral manifolds of $\eta$ in $U$ for $d \omega$-almost all $\omega \in \Omega$.

PROOF. If $R_{\omega}=\mathbf{H}^{m} \mathrm{~L} B_{\omega} \wedge \vec{R}_{\omega}$, then by Lemma C, $\vec{R}_{\omega}(x)$ is associated with the plane associated with $\eta$ for $\mathbf{H}^{m}$-almost all $x$.

If $B_{\omega}$ contains a $\mathbf{H}^{m}$ positive measure of points of a component of an integral manifold of $\eta$ in $U$ then, since $R_{\omega}$ has no boundary in $U, B_{\omega}$ has to contain $\mathbf{H}^{m}$ almost all of this component. Since $\mathbf{M}\left(R_{\omega} L U\right)<\infty, B_{\omega}$ has to be the union of at most countably many such components (modulo sets of $\mathbf{H}^{m}$-measure zero).

PROOF OF PROPOSITION 2. Define

$$
H(x, y, z)= \begin{cases}1 & \text { for } y>0 \\ 0 & \text { otherwise }\end{cases}
$$

and

$$
\xi(x, y, z)=e_{1} \wedge e_{2}+. H(x, y, z) e_{3} \wedge e_{2} .
$$

Then $N=L^{3} \wedge \xi$ is the desired example (actually, $N \in \mathbb{N}_{2}^{\text {loc }}\left(\mathbb{R}^{3}\right)$, but that is clearly unessential). In fact, $\xi L\{y>0\}$ is smooth and $\operatorname{div} \xi=0$ for $y>0$, so that it has an essentially unique mass decomposition by Lemma 7 :

$$
M_{s}^{1}=\mathbf{H}^{2} L\{(x, y, s): y<0\} \wedge\left(e_{1} \wedge e_{2}\right) .
$$

Similarly for $\xi L\{y<0\}$,

$$
M_{s}^{2}=\mathbf{H}^{2} L\{(x, y, x+s): y<0\}\left(e_{1}+e_{3}\right) \wedge e_{2}
$$

and

$$
\begin{aligned}
& \partial M_{s}^{1}=\mathbf{H}^{1} L\{(x, 0, s): x \in \mathbb{R}\} \wedge\left(-e_{1}\right), \\
& \partial M_{s}^{2}=\mathbf{H}^{1} L\{(x, 0, x+s): \mathbb{R}\} \wedge\left(e_{1}+e_{3}\right) .
\end{aligned}
$$

On the other hand,

$$
\partial N=-L^{3} \wedge \operatorname{div} \xi=-L^{3} \wedge\left(-e_{3}\right)_{\{y=0\}}^{\delta}=\mathbf{H}^{2} L\{(x, 0, z): z, x \in \mathbb{R}\} \wedge e_{3},
$$

and $N$ cannot be obtained as a convex integral of $M_{s}^{1}$ and $M_{s}^{2}$.

The next example shows that for codimensions greater than one, the smoothness assumption cannot be dropped in Proposition 1. 
Proposition 3. There exists a current $N \in \mathbb{N}_{2}\left(\mathbb{R}^{4}\right), \partial N=0$, and a set $X$, $\mathbf{H}^{2}(X)>0$, such that every $x \in X$ has no neighbourhood $U$ for which there exists a family $\left\{R_{s}\right\}_{s \in \mathbf{R}^{2}}$, satisfying (17)--(20) $(n=4, m=2)$.

PROOF. Let $\xi=e_{1} \wedge e_{2}+H e_{3} \wedge e_{2}$ be as in the proof of Proposition 2, and

$$
N=N_{0}-\partial N_{0} \times[0,1]+N_{0} \times\{1\},
$$

where $N_{0}=L^{3} \wedge \xi$, and $[0,1]$ is in $\mathbb{R}^{4}$ in the direction perpendicular to $\mathbb{R}^{3}$. We have $\partial N=0$. Let $x \in \operatorname{spt} N_{0}$, i.e. the $x-z$ plane in $\mathbb{R}^{3}$ and $U$ be an open ball around $x$. Suppose that for some such $U$

$$
\begin{aligned}
& N L U=\int_{\Omega} R_{\omega} d \omega, \quad R_{\omega} \in \mathbf{R}_{2}\left(\mathbb{R}^{4}\right), \\
& \|N L U\|=\int_{\Omega}\left\|R_{\omega}\right\| d \omega, \\
& \partial R_{\omega}=0
\end{aligned}
$$

then $R_{\omega} \mathrm{L} \mathbf{R}^{3}$ is a mass decomposition of $N_{0} \mathrm{~L}\left(U \cap \mathbb{R}^{3}\right)$, and we claim that it is also a boundary mass decomposition, which gives a contradiction in view of Proposition 2. We can assume that the radius of $U$ is less than one, and by Lemma $\mathrm{C}$

$$
\left.\left.R_{\omega} \mathrm{L}\left(\mathbb{R}^{3} \times\right] 0,1[\cap U)=\left(S_{\omega} \times\right] 0,1\right]\right)\llcorner U
$$

for some $S_{\omega} \in \mathbf{R}_{1}\left(\mathbb{R}^{4}\right)$ and spt $S_{\omega} \subset \mathbb{R}^{3}$. Also

$$
\begin{aligned}
& \left.\left.\left.\left.\int R_{\omega} L\left(\left(\mathbb{R}^{3} \times\right] 0,1\right]\right) \cap U\right) d \omega=\left(-\partial N_{0} \times\right] 0,1\right]\right) L U \\
& \left.\left.\left.\left.\int \| R_{\omega} L\left(\left(\mathbb{R}^{3} \times\right] 0,1\right]\right) \cap U\right)\|d \omega=\|\left(\partial N_{0} \times\right] 0,1\right]\right) L U \|,
\end{aligned}
$$

and hence

$$
\begin{aligned}
& \left.\left.\left.-\int\left(S_{\omega} \times\right] 0,1\right]\right)\left\llcorner U d \omega=\left(\partial N_{0} \times\right] 0,1\right]\right)\llcorner N \\
& \left.\left.\left.\int \|\left(S_{\omega} \times\right] 0,1\right]\right)\left\llcorner U\|d \omega=\| \partial N_{0} \times\right] 0,1\right]\llcorner U \|,
\end{aligned}
$$

what implies

$$
\begin{aligned}
& -\int S_{\omega} \mathrm{L}\left(U \times \mathbf{R}^{3}\right)=\partial N_{0} L\left(U \cap \mathbb{R}^{3}\right) \\
& \int\left\|S_{\omega} L\left(U \cap \mathbb{R}^{3}\right)\right\|=\left\|\partial N_{0} L\left(U \cap \mathbb{R}^{3}\right)\right\|
\end{aligned}
$$

Also

$$
\partial\left(R_{\omega} L\left(U \cap \mathbb{R}^{3}\right)\right)=-S_{\omega} L\left(U \cap \mathbb{R}^{3}\right),
$$

since $\partial R_{\omega} L U=0$. But then, (27) $\cdots(28)$ would imply that $R_{\omega} L \mathbb{R}^{3}$ is a simultaneous boundary mass decomposition of $N_{0} \mathrm{~L} U$. 


\section{BIBLIOGRAPHY}

[B] J. B. Brothers (editor), Some open problems in geometric measure theory, Geometric Measure Theory and the Calculus of Variations (W. K. Allard and F. J. Almgren, eds.), Amer. Math. Soc., Providence, R. I., 1986.

[F] H. Federer, Geometric measure theory, Springer-Verlag, Berlin and New York, 1969.

[FR] W. H. Fleming and R. Rishel, An integral formula for total gradiant variation, Arch. Math. II (1960), 218-222.

[HP] R. M. Hardt and J. T. Pitts, Solving plateau problem for hypersurfaces without compactness theorem for integral currents, Geometric Measure Theory and the Calculus of Variations, (W. K. Allard and F. J. Almgren, eds.), Amer. Math. Soc., Providence, R. I., 1986.

[H] R. Harvey, Introduction to geometric measure theory, preprint.

[M] F. Morgan, On finiteness of the number of stable minimal hypersurfaces with a fixed boundary, Indiana Univ. Math. J. 35 (1986), 779-834.

Department of MAthematics, Massachusetts Institute of Technology, CAMBRIDGE, MASSACHUSETTS 02139 